University of Wollongong

Research Online

Australian Institute for Innovative Materials -

Papers

Australian Institute for Innovative Materials

$1-1-2014$

\title{
Three-dimensional printing fiber reinforced hydrogel composites
}

Shannon Bakarich

University of Wollongong, seb798@uowmail.edu.au

Robert Gorkin III

University of Wollongong, rgorkin@uow.edu.au

Marc in het Panhuis

University of Wollongong, panhuis@uow.edu.au

Geoffrey M. Spinks

University of Wollongong, gspinks@uow.edu.au

Follow this and additional works at: https://ro.uow.edu.au/aiimpapers

Part of the Engineering Commons, and the Physical Sciences and Mathematics Commons

Research Online is the open access institutional repository for the University of Wollongong. For further information contact the UOW Library: research-pubs@uow.edu.au 


\title{
Three-dimensional printing fiber reinforced hydrogel composites
}

\begin{abstract}
An additive manufacturing process that combines digital modeling and 3D printing was used to prepare fiber reinforced hydrogels in a single-step process. The composite materials were fabricated by selectively pattering a combination of alginate/acrylamide gel precursor solution and an epoxy based UVcurable adhesive (Emax $904 \mathrm{Gel}-\mathrm{SC}$ ) with an extrusion printer. UV irradiation was used to cure the two inks into a single composite material. Spatial control of fiber distribution within the digital models allowed for the fabrication of a series of materials with a spectrum of swelling behavior and mechanical properties with physical characteristics ranging from soft and wet to hard and dry. A comparison with the "rule of mixtures" was used to show that the swollen composite materials adhere to standard composite theory. A prototype meniscus cartilage was prepared to illustrate the potential application in bioengineering.
\end{abstract}

\section{Keywords}

hydrogel, reinforced, composites, fiber, three, printing, dimensional

Disciplines

Engineering | Physical Sciences and Mathematics

\section{Publication Details}

Bakarich, S. E., Gorkin, III, R., in het Panhuis, M. \& Spinks, G. M. (2014). Three-dimensional printing fiber reinforced hydrogel composites. ACS Applied Materials and Interfaces, 6 (18), 15998-16006. 


\title{
3D printing fibre reinforced hydrogel composites
}

Shannon E. Bakarich ${ }^{1}$, Robert Gorkin (III) ${ }^{1}$, Marc in het Panhuis ${ }^{1,2}$ and Geoffrey M. Spinks ${ }^{1,3} *$

${ }^{1}$ Intelligent Polymer Research Institute, ARC Centre of Excellence for Electromaterials Science, AIIM Facility, University of Wollongong, NSW 2522, Australia

${ }^{2}$ Soft Materials Group, School of Chemistry, University of Wollongong, NSW 2522, Australia

${ }^{3}$ School of Mechanical, Materials and Mechatronic Engineering, University of Wollongong, NSW 2522, Australia

\begin{abstract}
An additive manufacturing process that combines digital modeling and 3D printing was used to prepare fibre reinforced hydrogels in a single-step process. The composite materials were fabricated by selectively pattering a combination of alginate/acrylamide gel precursor solution and an epoxy based UV-curable adhesive (Emax 904 Gel-SC) with an extrusion printer. UV irradiation was used to cure the two inks into a single composite material. Spatial control of fibre distribution within the digital models allowed for the fabrication of a series of materials with a spectrum of swelling behaviour and mechanical properties with physical characteristics ranging from soft and wet to hard and dry. A comparison with the 'rule of mixtures' was used to show that the swollen composite materials adhere to standard composite theory. A prototype meniscus cartilage was prepared to illustrate the potential application in bioengineering.
\end{abstract}

\section{KEYWORDS:}


3D printing, alginate/polyacrylamide hydrogel, artificial meniscus, composite hydrogel, Rule of Mixtures

\section{INTRODUCTION}

Three dimensional (3D) printing is an additive manufacturing process to construct 3D objects from a digital model that has received a great deal of attention from a diverse range of fields ${ }^{1}$ including electronics ${ }^{2}$, regenerative medicine ${ }^{3-4}$, aerospace ${ }^{2}$ and microfluidics ${ }^{5}$. Objects are made by sequentially depositing layer upon layer of ink to build up the 3D structure. ${ }^{1}$ An advantage of 3D printing over other additive fabrication technologies is that multiple materials can be incorporated into a single build. With digital modeling this feature gives the printer spatial control of the material properties within an object. Gradient structures and novel composite materials can be printed by selectively patterning different inks. ${ }^{5-7}$ These processing advantages are particularly useful for the construction of complex structures where spatially varying mechanical properties are likely needed. A primary example of such an area which utilizes such attributes is bioengineering, where printing composites is required to mimic realistic tissues like skin or even organs.

Recent studies have examined the use of hydrogels for 3D printing soft tissue applications. ${ }^{8-12}$ The preparation of complex 3D shapes from conventional ${ }^{13}$ and tough ${ }^{14-15}$ hydrogels has been demonstrated. These studies have generally been limited to printing a single ink to produce objects of uniform composition. Fibre-reinforced hydrogel composites have also been fabricated through 3D printing in a two-step process. First the fibre scaffold was printed and the composite structure is completed by immersing the scaffold into a hydrogel precursor solution and polymerizing the gel around the fibres. ${ }^{16-17}$ These studies and others involving hydrogels reinforced with fibres, ${ }^{18}$ and woven textiles ${ }^{19-20}$ have demonstrated an increase in modulus and strength. Indeed, many soft tissues can be thought of as fibre reinforced hydrogel composites. ${ }^{16}$ For example, articular cartilage is a proteoglycan gel matrix reinforced with collagen fibres with strength varying from 1.5 to 30 MPa depending on fibre concentration. ${ }^{21}$ 
The present study investigated the preparation of fibre-reinforced hydrogels where both fibre reinforcement and matrix were 3D printed in a single-step process. Based on previous work demonstrating extrusion printing coupled with ultra-violet (UV) radiation induced gelation of a hybrid ionic-covalent entanglement (ICE) gel $^{14}$, the method was further developed to incorporate UV cured rigid epoxy reinforcement. A series of uniaxially oriented, continuous fibre reinforced hydrogels was prepared and the mechanical properties and swelling behaviour evaluated. The effect of fibre volume fraction on the elastic modulus was analysed using standard composites theory. In addition, a prototype articular meniscus cartilage was printed that mimicked both the complex shape and internal fibre-reinforced structure of the natural system.

\section{EXPERIMENTAL SECTION}

\section{Materials}

All materials were used as received and all solutions were prepared using Milli-Q water (resistivity $=18.2 \Omega \mathrm{cm}$ ). $\alpha$-Keto glutaric acid photo-initiator was purchased from Fluka (Australia). Acrylamide solution, 40\%, for electrophoresis, sterile-filtered, alginic acid sodium salt, from brown algae with Brookfield viscosity ( $2 \%$ in $\mathrm{H}_{2} \mathrm{O}$ at $25{ }^{\circ} \mathrm{C}$ ) of $250 \mathrm{mPa}$ s, N,N'-methylenebisacrylamide, calcium chloride (minimum 93.0\% granular anhydrous) cross linkers and ethylene glycol rheology modifier were purchased from Sigma Aldrich (Australia). A commercial epoxy based UV-curable adhesive (Emax 904 Gel-SC) was purchased from Ellsworth Adhesives (Australia).

\section{Hydrogel ink formulations}

The ICE hydrogel ink was prepared using a method previously published. ${ }^{14}$ The ink was prepared by dissolving $4.50 \mathrm{ml}$ of acrylamide, $38.7 \mathrm{mg}$ of N,N'-methylenebisacrylamide and $36.9 \mathrm{mg}$ of $\alpha$ keto glutaric acid in $5.25 \mathrm{ml}$ of Milli-Q water, $1.5 \mathrm{ml}$ of the $0.1 \mathrm{M}$ calcium chloride stock solution and $3.75 \mathrm{ml}$ of ethylene glycol. The latter was used as a non-volatile co-solvent with the ratio of ethylene glycol to water fixed at 1:2.5. A spatula was then used to dissolve $450 \mathrm{mg}$ of alganic sodium salt into the printing solution. 
An ink for printing a dissolvable support structures was prepared by mixing $1.5 \mathrm{ml}$ of the $0.1 \mathrm{M}$ calcium chloride stock solution and $3.75 \mathrm{ml}$ of ethylene glycol in $9.75 \mathrm{ml}$ of of Milli-Q water. A spatula was then used to dissolve $450 \mathrm{mg}$ of alganic sodium salt into the printing solution.

\section{Rheology}

The flow behaviour of each of the inks was determined using a AR2G Rheometer coupled with a cone and plate measuring system (diameter $40 \mathrm{~mm}$, angle $2^{0} 06^{\prime}$ and truncation $55 \mu \mathrm{m}$ ) and a heat controlled sample stage (TA Instruments Peltier plate). The viscosity was measured as a function of shear rate $\left(1-500 \mathrm{~s}^{-1}\right)$ at $25{ }^{0} \mathrm{C}$. All experimental studies were repeated in quadruplicate and the error was determined using standard deviation.

\section{Contact angle}

The contact angle of the extrusion inks on the printing substrate was determined using a Dataphysics Contact Angle System OCA. A square of polypropylene sheet was attached to the sample stage and a drop of the ink was placed onto the substrate. SCA20 software was used to calculate the angle between the droplet and the substrate at 1 min intervals for 5 min.

\section{Extrusion printing}

A 3D-Bioplotter system (EnvisionTEC, Germany) was fashioned with a commercial UV-curing system to print hydrogel composite structures from the alginate/acrylamide ink, the Emax 904 GelSC adhesive and the alginate support gel. A Dymax BlueWave 75 Rev 2.0 UV Light Curing Spot Lamp System using a 19+ W/cm ${ }^{2}$ UV source with a 1 meter light guide was used to cure the printed ink. The end of the light guide was fixed to a custom made UV safety shield that covered the Bioplotter's build area. A slot in the UV safety box allowed the UV spot to be positioned directly over the patterned ink.

Computer-aided design (SolidWorks) was used to construct digital models of the tensile specimens and artificial meniscus structures that were to be printed. The composite materials were designed by creating an assembly from separate parts as illustrated in Figure 1 for the tensile specimens. EnvisionTEC software was used to slice the digital model into a stack of two 
dimensional layers (250 $\mu$ m thick) to determine the print path. Material files and internal structures were then added to each part within the assembly. Tensile samples were designed with systematically varying volume fraction of reinforcing Emax fibres. The fibre diameter and length were kept constant and the number of fibres varied to achieve the desired volume fraction.

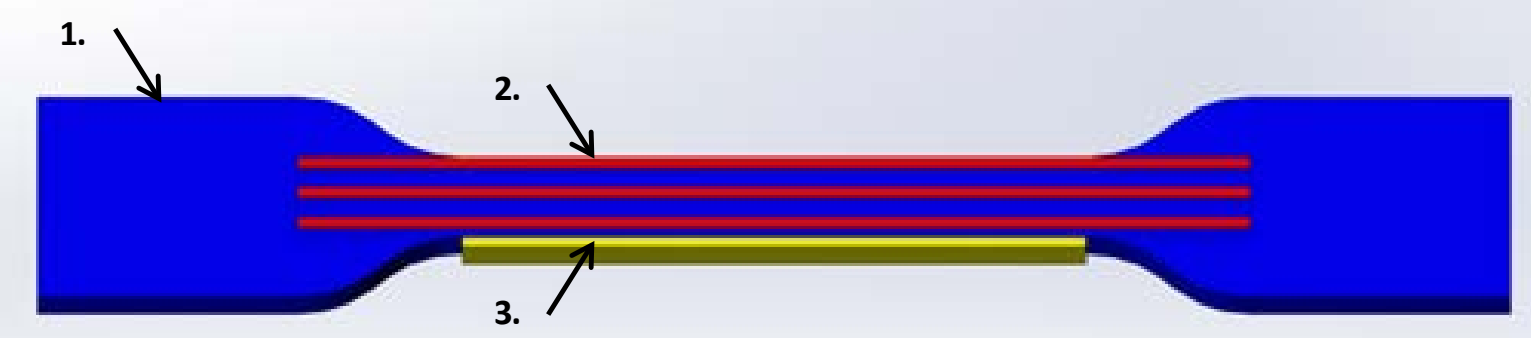

Figure 1. A typical example of a CAD assembly model for a $62.5 \%$ Emax hydrogel composite tensile specimen. Numbers 1 and 2 represent the Emax and ICE gel constituents, respectively, and number 3 represents a printed support structure (alginate gel) that is removed after printing.

The alginate ICE gel ink, the Emax 904 Gel-SC adhesive and the alginate support gel ink were loaded into the 3D-Bioplotter in separate pressurised syringe barrels in the temperature controlled print heads. 23 gauge syringe tips (diameter $0.337 \mathrm{~mm}$ ) were fitted to the ends of each of the barrels. The barrel temperature was held stable during printing at $25{ }^{0} \mathrm{C}$. The hydrogel ink formulation was printed at 0.7 bar and with head speed of $5 \mathrm{~mm} / \mathrm{s}$. The Emax $904 \mathrm{Gel}-\mathrm{SC}$ adhesive was printed at of 0.9 bar and with head speed of $5 \mathrm{~mm} / \mathrm{s}$. The alginate support gel ink was printed at of 0.6 bar and with head speed of $5 \mathrm{~mm} / \mathrm{s}$. The UV light was passed over each layer of ink for $50 \mathrm{~s}$ and the completed structure for 200 s. A paper wipe was used to carefully remove the soft alginate support gel from the completed specimen.

The composite structures were printed onto squares cut out of polypropylene sheets attached to the build stage. The poly(pyropylene) sheets acted as release layers allowing easy separation of the printed material from the build stage. Hydrogel composites were printed with nominal Emax volume fraction of $0,25,37.5,50,62.5,75,87.5$ and $100 \%$. All tensile specimens were printed into 
dumbbell shapes with Emax end grips for ease of clamping. Printed materials were characterised immediately after the completion of the curing process.

\section{Imaging}

The print quality of the hydrogel composites was examined with a Leica M205 A microscope. A single drop of red food dye was added to the hydrogel ink before printing to identify the hydrogel phase. Cross-sectional images were obtained from strips of the hydrogel composites cut into $1 \mathrm{~mm}$ slices with a razor blade. Leica Application Suite V4 software was then used to measure the cross sectional area of the printed hydrogel composites. The true Emax volume fraction of the printed composites was calculated as the ratio between the cross sectional area of Emax and the total cross sectional area. Four repeat tests were performed on each type of composite sample with errors estimated from one standard deviation.

\section{Swelling}

The swelling ratio was determined after immersing printed hydrogel composite samples in $0.1 \mathrm{M}$ calcium chloride (72 hr) to fully cross link the alginate followed by immersion in Milli-Q water (72 hr). A single drop of red food dye was added to the Milli-Q water to aid in imaging the swollen hydrogels. Swelling ratios ' $Q$ ’ were calculated using:

$$
Q=\frac{W_{S}-W_{D}}{W_{D}} \times 100 \%,(1)
$$

where ' $\mathrm{W}_{\mathrm{D}}$ ' is the dry weight of hydrogel and ' $\mathrm{W}_{\mathrm{S}}$ ' is the swollen mass of the hydrogel. Samples were dried by being placed in an oven at $60{ }^{\circ} \mathrm{C}$ for $72 \mathrm{hr}$.

\section{Mechanical testing}

The mechanical properties of the printed composites were determined using a Shimadzu EZ-L Universal Mechanical Tester. Tensile tests were performed using a $50 \mathrm{~N}$ load cell for low modulus/strength samples, and a $500 \mathrm{~N}$ load cell for high modulus/strength samples. Printed tensile specimens were strained to failure at a rate of $10 \mathrm{~mm} / \mathrm{min}$. The applied stress was calculated using the average cross sectional area of the unstrained specimen at the gauge length. The failure stress and failure strain were calculated from the onset of failure. The Work of Extension was calculated 
as the area under the stress-strain curve to failure. Four repeat tests were performed on each type of composite sample with errors estimated from one standard deviation.

An adhesion test was performed to determine the strength of the bond between the ICE gel matrix and Emax fibres in the printed composites. A tensile specimen was printed with Emax ends and a band of ICE gel. The tensile specimen was extended in the direction perpendicular to ICE gel band until failure. The position of failure was noted in each of these tests.

\section{RESULTS AND DISCUSSION}

The aim of the present study was to evaluate the effectiveness of fibre-reinforcement of 3D printed hydrogel structures where both the fibre reinforcement and the matrix were printed in a single process. Initially Emax 904 Gel-SC adhesive was identified as a material with suitable UV cure characteristics, appropriate rheological properties for extrusion printing, adhesion to the alginate ICE gel and acceptable mechanical properties for fibre reinforcement. Utilising a multibarreled extrusion printer, dumbbell shaped tensile specimens were printed and cured with UV radiation to demonstrate composite printability and to measure mechanical properties.

\section{Extrusion printing}

The viscosity ' $\eta$ ' of the Emax and alginate support gel inks was measured to determine the optimal conditions for extrusion printing. The observed viscosity data (Figure 2a) were fitted to a power law model ${ }^{22}$ :

$$
\eta=K \dot{\gamma}^{n-1}
$$

where ' $\gamma$ ' is the shear rate, ' $K$ ' is the consistency index and ' $n$ ' is the power law index. Both the Emax and alginate support gel inks display shear-thinning flow behaviour $(n<1)$ similar to the previously published alginate ICE gel ink. ${ }^{14}$ In that previous rheological study it was determined that our extrusion printing system in conjunction with a 23 gauge syringe tip could process inks with consistency index values between 20 and $150 \mathrm{~Pa} \mathrm{~s}^{\mathrm{n}}$ and power law index values between 0.35 and $0.45 .^{14}$ The alginate ICE gel ink has a consistency index $80 \pm 10 \mathrm{~Pa} \mathrm{~s}^{\mathrm{n}}$ and power law index 
$0.37 \pm 0.01$ (Table 1 ). The new inks were also found to have flow characteristics compatible for extrusion printing with consistency index and power law index values of $41 \pm 1 \mathrm{~Pa} \mathrm{~s}^{\mathrm{n}}$ and $0.44 \pm$ 0.01, respectively, for Emax and $50 \pm 10 \mathrm{~Pa} \mathrm{~s}^{\mathrm{n}}$ and $0.38 \pm 0.02$, respectively, for the alginate support ink.

The contact angle between the poly(propylene) sheet and each of the extrusion inks was measured over a 5 minute period (Figure $2 b$ ) to determine their wetting behaviour. The contact angle indicates whether the ink will spread on the substrate or bead up in the time period between printing and curing of the ink. Wetting is an important printing parameter because the accuracy of a printer is limited by the extent of which an ink will spread once deposited. After 5 minutes the Emax had a lower contact angle $\left(63 \pm 3^{\circ}\right)$ and hence larger degree of wetting than the alginate ICE gel $\left(88 \pm 6^{\circ}\right)$ and the alginate support gel $\left(90 \pm 3^{\circ}\right)$. 

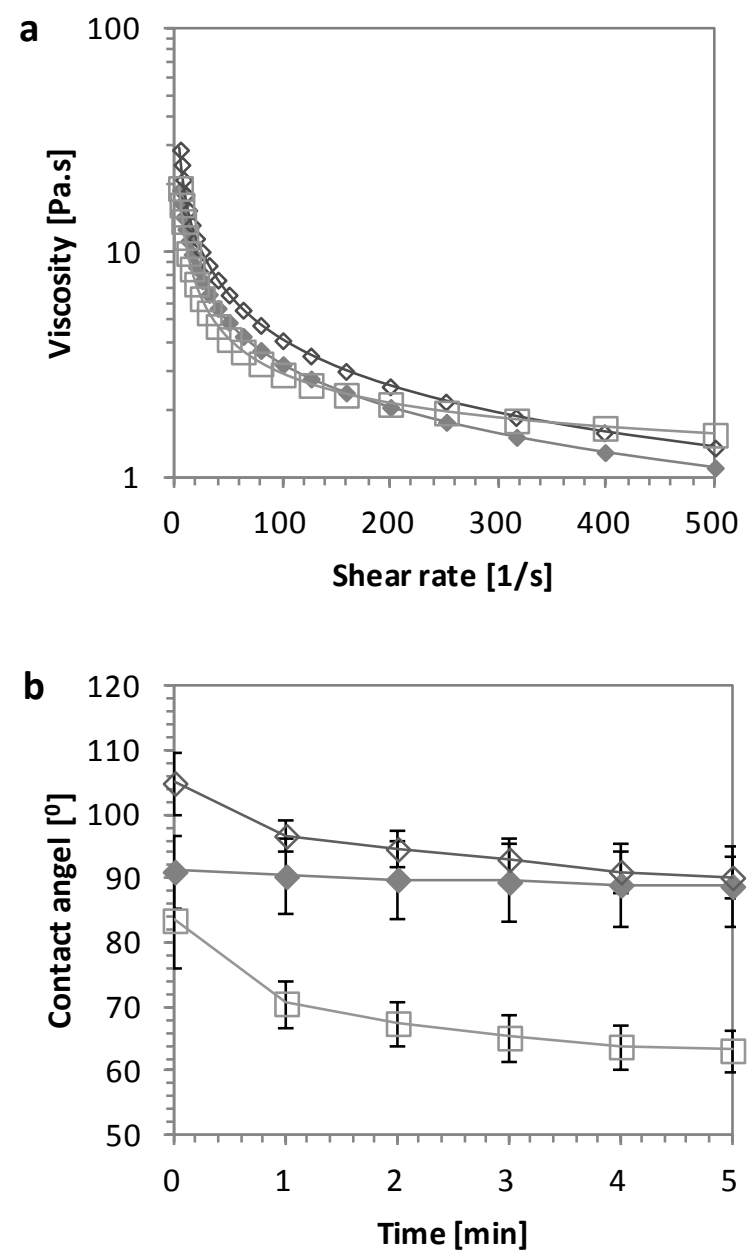

Figure 2. a. Experimental viscosity data (symbols) and flow curves fitted to equation (2) for Emax (open squares), alginate support gel (closed diamonds) and alginate ICE gel (open diamonds). b. Contact angle measurements of Emax (open squares), alginate support gel (closed diamonds) and alginate ICE gel (open diamonds) on the poly(propylene) sheet measured as a function of time.

Table 1. Summary of the rheological parameters measured for each material. ${ }^{a}$

\begin{tabular}{lcl}
\hline Material & $\mathrm{K}\left[\mathrm{Pa} \mathrm{s}^{\mathrm{n}}\right]$ & $\mathrm{n}$ \\
\hline Emax & $41 \pm 1$ & $0.44 \pm 0.01$ \\
& & \\
Alginate support gel & $50 \pm 10$ & $0.38 \pm 0.02$ \\
Alginate ICE gel & $80 \pm 10$ & $0.37 \pm 0.01$
\end{tabular}

\footnotetext{
${ }^{\mathrm{a}}$ Consistency (K) and power law (n) indices were obtained through curve fitting with Equation 2.
} 
Fibre reinforced hydrogel composites were fabricated with a commercially available 3D printer and cured with a UV light source. Initially the composite structures were printed with the Emax and alginate ICE gel inks. The patterned ink was cured after the entire structure had been printed. In Figure 3 cross sections of the printed composites were compared with cross sections of their CAD model (Figure 3a \& d) to assess the accuracy of this printing method. Two types of defects were noted. Firstly, examination of the exposed Emax edges revealed that the Emax ink was spreading once patterned, this can be attributed to its low contact angle. This surface wetting produced a tapered edge rather than the desired square shape (Figure 3b). A more defined vertical edge (Figure 3c) was achieved by using a removable alginate support ink at the edges to prevent spreading of Emax. The support ink was not UV-curable and was easily removed after printing. Secondly, inspection of the fibre structure of the composite hydrogels (Figure 3e) revealed that the fibres were collapsing prior to curing when UV exposure was performed after the printing of several layers. A better defined fibre structure (Figure $3 f$ ) that was closer to the CAD model was achieved by partially curing each layer of ink for $50 \mathrm{~s}$ during the fabrication process. After analysing these results all further fibre reinforced hydrogel composite structures were printed with an alginate support ink mould against all exposed Emax edges and each layer of ink was partially cured prior to printing the following layer. 

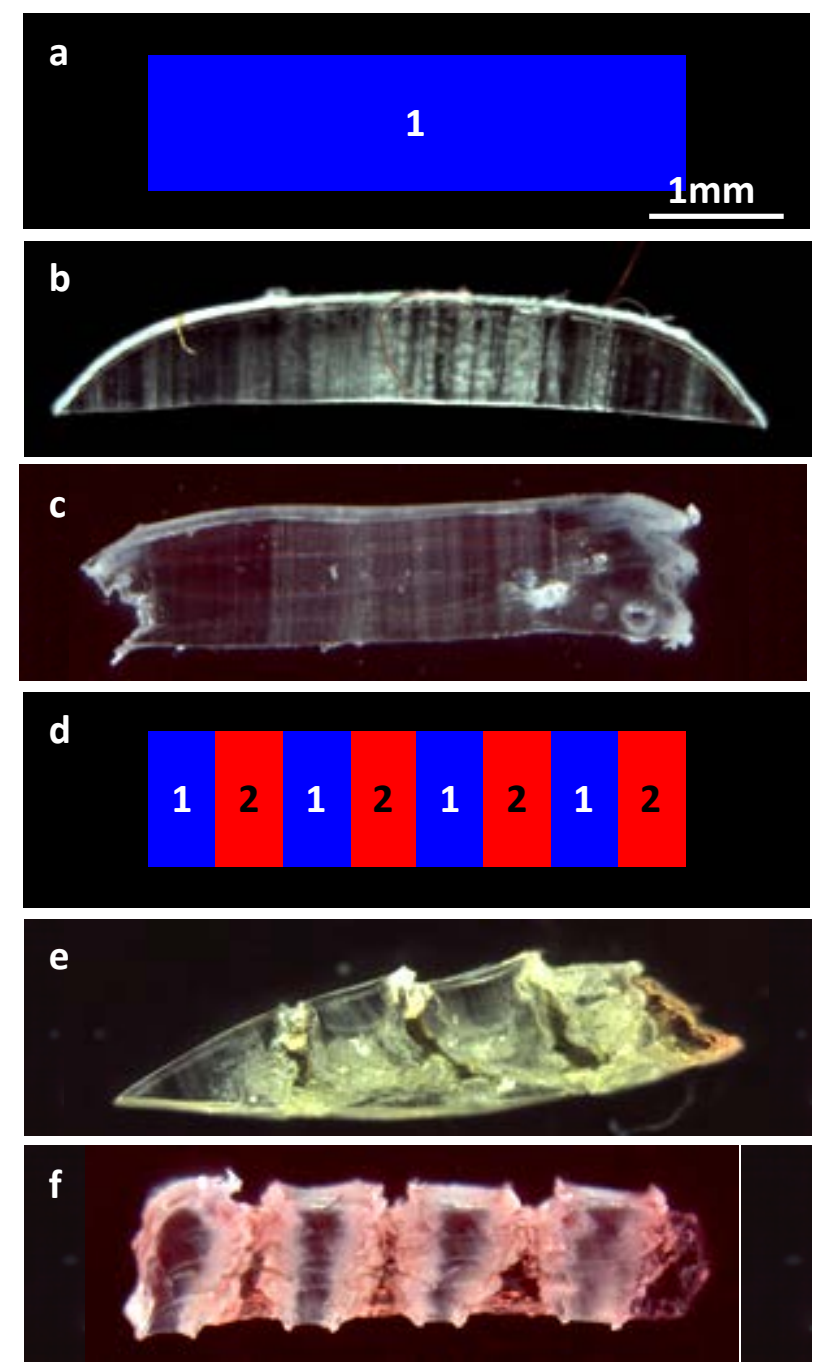

Figure 3. a. The cross section of a CAD model of a 100\% Emax sample compared to microscope images of the edge of $100 \%$ Emax samples printed b. with no support gel mould and c. with a support gel mould. d. The cross section of a CAD model of a 50\% Emax sample compared to microscope images of 50\% Emax samples e. cured after printing 4 layers of ink and f. cured after printing each layer of ink. In the CAD models component 1 defines Emax and component 2 defines ICE gel. For the microscope images red food dye was used to highlight the ICE gel.

Cross sections of the fiber reinforced hydrogels were also used to calculate the true Emax volume fraction of the printed composites. The true volume fraction was calculated as the ratio between the cross sectional area of Emax and the total cross sectional area of the printed composite. Figure 4 is a plot of the true Emax volume fraction against the Emax volume fractions of the CAD models. The plot shows that the true Emax volume fraction is greater than the intended Emax for all 
of the printed hydrogel composites. This result indicates the Emax was deposited through the printer at a higher rate than the ICE gel. In the following the true Emax volume fraction is used to identify each of the fiber reinforced hydrogel composites.

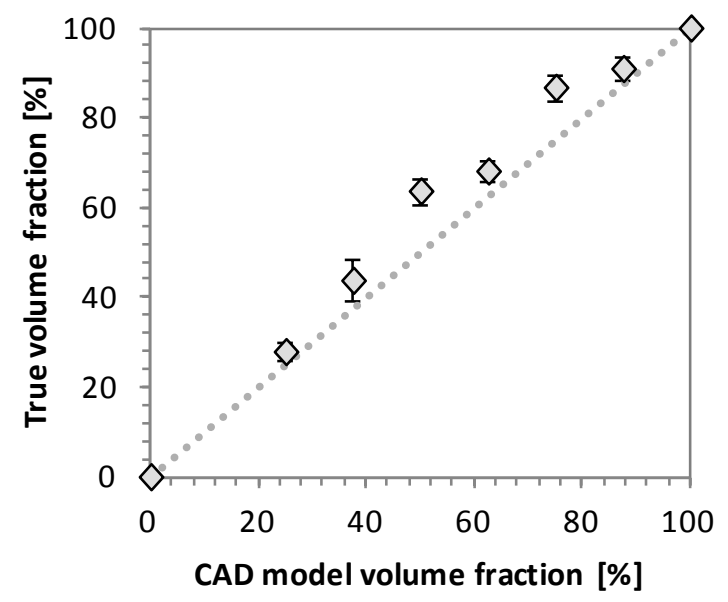

Figure 4 The true Emax volume fraction calculated from the cross sections microscope images as a function of the volume fraction from the CAD models.

\section{Swelling ratio and mechanical testing of fully swollen hydrogel composites}

Hydrogel mechanical properties are strongly dependent upon swelling ratio which is in turn influenced by the environment. The swelling ratio and mechanical properties of the printed hydrogel composites were characterised when fully swollen because hydrogel materials are most commonly implemented in aqueous environments. Fully swollen hydrogel composites were prepared by immersing the as-printed composites in $0.1 \mathrm{M}$ calcium chloride for 72 hrs to crosslink the alginate polymer network and to enhance their mechanical properties. ${ }^{14}$ The hydrogels were then allowed to equilibrate in water for a further 72 hrs. Based on the mass loss during heating at $60^{\circ} \mathrm{C}$ for $72 \mathrm{~h}$ to fully dry the hydrogel the swelling ratios were determined using equation (1). The ICE gel exhibited a swelling ratio of $350 \pm 30 \%$ and the Emax exhibited a swelling ratio of $19 \pm 3$ \%. As expected, the swelling ratios of hydrogel composites decreased as the Emax volume fraction was increased. The plot in Figure 5 demonstrates that the swelling ratios of the composites ' $Q_{c}$ ' closely approximates the simple rule of mixtures for swelling ratio which was calculated using: 


$$
Q_{c}=Q_{f} V_{f}+Q_{m} V_{m},
$$

where ' $Q_{f}$ ' and ' $Q_{m}$ ' are the swelling ratio of the fibre (Emax) and the matrix (ICE gel) respectively and ' $V_{f}$ ' and ' $V_{m}$ ' are the fibre and matrix volume fractions, respectively. The rule of mixtures is a weighted mean which assumes that each component's contribution to the total swelling ratio is additive.

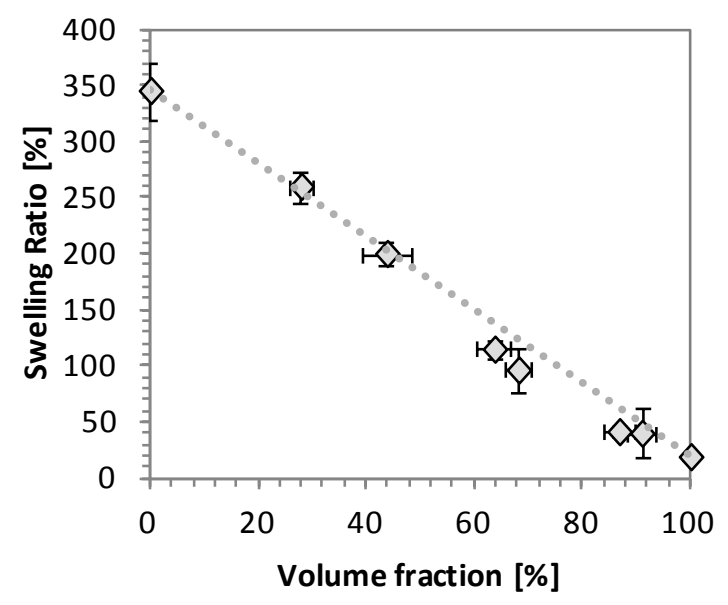

Figure 5. The swelling ratio of swollen hydrogel composites measured as a function of Emax volume fraction. The broken line indicates the rule of mixtures (Equation 3).

Tensile tests on the swollen hydrogel composites in the fibre direction were performed to determine the effect of the Emax volume fraction on the composites' mechanical properties. Figure 6a - d includes photographs of the hydrogel composite tensile specimens when clamped in the tensile tester. Examples of typical stress-strain curves of the swollen fibre reinforced hydrogels are shown in Figure $6 \mathrm{~g}$ and a summary of the mechanical data is included in Table 2. The ICE gel exhibited an elastic modulus of $260 \pm 20 \mathrm{kPa}$ and the Emax had an elastic modulus of $2700 \pm 200$ $\mathrm{kPa}$. The ICE gel is soft and wet whilst the Emax is hard and dry. The tensile strength of the $100 \%$ ICE gel was $80 \pm 10 \mathrm{kPa}$ with a failure strain of $48 \pm 4 \%$ compared with a tensile strength of $2400 \pm$ $100 \mathrm{kPa}$ and a failure strain of $230 \pm 10 \%$ for the $100 \%$ Emax. The printed composite structures display a combination of properties in between these two extremes with an increase in elastic modulus, failure strength, failure strain and work of extension corresponding with an increase in 
Emax volume fraction and decrease in swelling ratio. By varying the Emax volume fraction of the CAD models the mechanical properties of the printed composites can be varied over several orders of magnitude.

To determine the efficiency of printed Emax fibres for reinforcing printed ICE gel, the experimental mechanical data was compared with standard composite theory. Maximum fibre reinforcement requires a perfect bond between matrix and fibre reinforcement so that the matrix and the fibre reinforcement achieve equal strains. An adhesion test was performed upon an ICE gel / Emax tensile specimen (Figure 6e) to determine the strength of the bond between the ICE gel matrix and Emax fibres in the printed composites. Figure $6 \mathrm{f}$ shows that mechanical failure occurred within the hydrogel band of the tensile specimen and not at the ICE gel / Emax interface indicating that the bond between the ICE gel and Emax is stronger than ICE gel. Due to the strength of this bond it can be assumed that there is no slipping between the matrix and fibre reinforcement so that equal strain is observed by the matrix and the fibre reinforcement when under tension.

When the strain between the matrix and fibre reinforcement is equal the fibre reinforcement experiences a greater stress than the matrix because the fibre reinforcement has a larger elastic modulus than the matrix. Based on this condition, the upper bound elastic modulus ' $E_{\|}$' of a perfectly reinforced composite was calculated using the rule of mixtures: ${ }^{23}$

$$
E_{\|}=E_{f} V_{f}+E_{m} V_{m}
$$

Where ' $E_{f}$ ' and ' $E_{m}$ ' are the elastic modulus of the fibre and the matrix, respectively. The rule of mixtures assumes that both the matrix and fibres behave linear elastically.

Ineffective fibre reinforcement occurs when stress from the matrix is not transferred to the fibre reinforcement; which could be caused by slipping between the matrix and fibre reinforcement, fibre end effects or a geometry off-set. Minimum fibre reinforcement occurs when a tensile load is applied perpendicular to the fibre direction. In this case the load is shared uniformly across the composite so that the stress in the fibres is equal to the stress in the matrix. Based on this condition, 
the lower bound elastic modulus ' $E_{\perp}$ ' of an ineffectively reinforced composite was calculated using the transverse rule of mixtures: ${ }^{23}$

$$
E_{\perp}=\frac{E_{f} E_{m}}{E_{f} V_{m}+E_{m} V_{f}} .
$$

The measured elastic modulus of the swollen hydrogel composites was compared with the rule of mixtures in Figure 6h. This plot demonstrates that printed Emax fibres provide effective reinforcement to the printed ICE gel and adhere to the upper bound rule of mixtures as the experimental values for elastic modulus very closely match the theoretical values calculated from Equation 4.
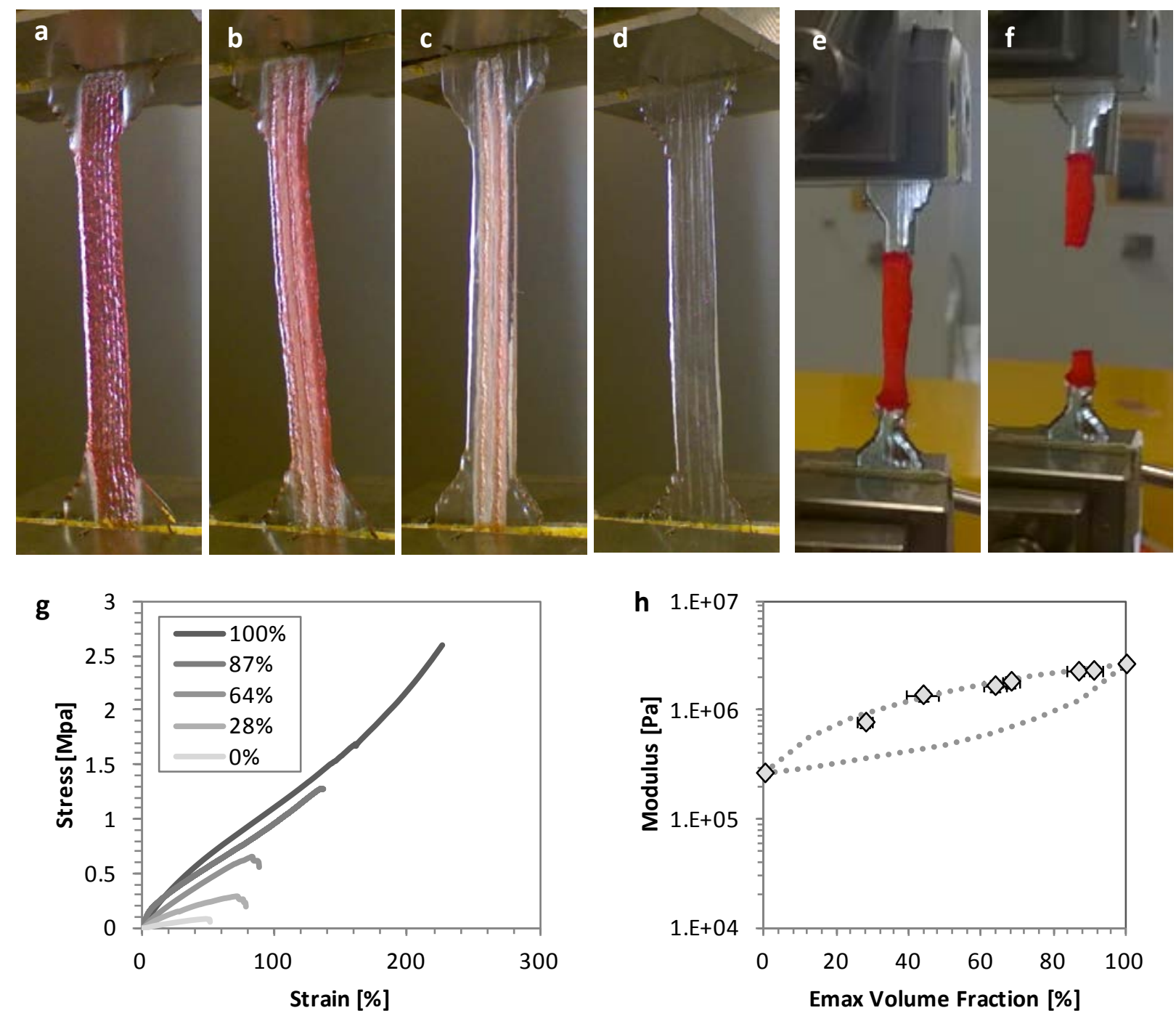

Figure 6. Photographs of 3D printed hydrogel composite tensile specimens with (a) 0, (b) 28, (i) 64 and (j) 100\% Emax volume fractions clamped for tensile testing. Photographs of ICE gel / Emax 
adhesion test sample (e) before and (f) after mechanical failure. For the photographs red food dye was used to highlight the ICE gel. g. Typical stress strain curves for swollen hydrogel composites with 0, 28, 64, 87 and 100\% Emax volume fractions. h. Mechanical properties of fully swollen hydrogel composites as a function of Emax volume fraction elastic modulus including the theoretical upper (Equation 4) and lower (Equation 5) bounds for composite elastic modulus.

Table 2 Summary of the mechanical properties of swollen hydrogel composites. ${ }^{\mathrm{b}}$

\begin{tabular}{|c|c|c|c|c|c|c|}
\hline $\begin{array}{l}\text { Emax } \\
\text { Volume } \\
\text { Fraction [\%] }\end{array}$ & $\begin{array}{l}\text { Elastic } \\
\text { Modulus } \\
{[\mathrm{MPa}]}\end{array}$ & $\begin{array}{l}\text { Upper } \\
\text { Limit } \\
{[\mathrm{MPa}]}\end{array}$ & $\begin{array}{l}\text { Lower } \\
\text { Limit } \\
{[\mathrm{MPa}]}\end{array}$ & $\begin{array}{l}\text { Failure } \\
\text { Stress } \\
{[\mathrm{MPa}]}\end{array}$ & $\begin{array}{l}\text { Failure } \\
\text { Strain } \\
{[\%]}\end{array}$ & $\begin{array}{l}\text { Work of } \\
\text { Extension } \\
{[\mathrm{kJ} / \mathrm{m} 3]}\end{array}$ \\
\hline 0 & $0.26 \pm 0.02$ & 0.26 & 0.26 & $\begin{array}{l}0.08 \\
0.01\end{array}$ & $48 \pm 4$ & $22 \pm 6$ \\
\hline $28 \pm 2$ & $0.77 \pm 0.07$ & 0.86 & 0.34 & $\begin{array}{l}0.31 \\
0.04\end{array}$ & $74 \pm 6$ & $140 \pm 20$ \\
\hline $43 \pm 5$ & $1.4 \pm 0.2$ & 1.2 & 0.40 & $\begin{array}{l}0.51 \\
0.05\end{array}$ & $81 \pm 8$ & $243 \pm 50$ \\
\hline $64 \pm 3$ & $1.7 \pm 0.2$ & 1.5 & 0.48 & $0.65 \pm 0.01$ & $89 \pm 8$ & $320 \pm 30$ \\
\hline $68 \pm 2$ & $1.9 \pm 0.2$ & 1.8 & 0.61 & $1.0 \pm 0.10$ & $110 \pm 6$ & $580 \pm 70$ \\
\hline $87 \pm 3$ & $2.2 \pm 0.1$ & 2.1 & 0.81 & $\begin{array}{l}1.30 \\
0.03\end{array}$ & $140 \pm 3$ & $1000 \pm 50$ \\
\hline $91 \pm 2$ & $2.3 \pm 0.1$ & 2.4 & 1.3 & $\begin{array}{l}1.66 \\
0.04\end{array}$ & $170 \pm 10$ & $1500 \pm 30$ \\
\hline 100 & $2.7 \pm 0.2$ & 2.7 & 2.7 & $\begin{array}{l}2.40 \\
0.10\end{array}$ & $230 \pm 10$ & $2800 \pm 200$ \\
\hline
\end{tabular}

\footnotetext{
${ }^{b}$ Upper limit and lower limit indicate elastic modulus calculations using equations 4 and 5, respectively.
}

\section{Swelling ratio and mechanical testing of as-printed hydrogel composites}

As 3D-Bioprinting has been touted for its ability to produce parts "as needed," it is important to understand the mechanical properties of the as-printed hydrogel composites to assess potential 
applications for immediate use. The as-printed ICE gel exhibited a swelling ratio of $260 \pm 30 \%$ and the Emax had a swelling ratio of $5 \pm 1 \%$. These two swelling ratios are less than the swelling ratios of the swollen materials which indicates that both the Emax and the ICE gel absorb water when exposed to an aqueous environment. Figure 7 demonstrates that the as-printed composite materials adhere to the law of mixtures for swelling (Equation 3) as expected.

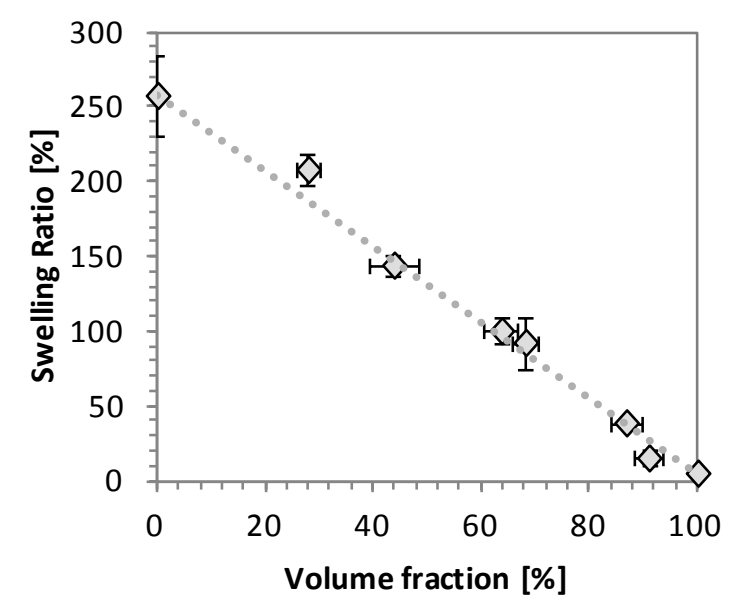

Figure 7. The swelling ratio of as-printed hydrogel composites measured as a function of Emax volume fraction. The dotted line indicates the rule of mixtures (Equation 3).

The mechanical characteristics of the as-printed materials differ drastically to the mechanical characteristics of the swollen composites. Examples of typical stress-strain curves for the as-printed reinforced hydrogels are shown in Figure 8a and a summary of the mechanical data is included in Table 3. The elastic modulus of the as-printed ICE gel (70 $\pm 4 \mathrm{kPa}$ ) is significantly lower than the elastic modulus of the swollen hydrogel because the as-printed alginate network is not cross linked until it is treated with calcium chloride. The Emax elastic modulus (76 $\pm 9 \mathrm{MPa})$ is significantly larger than the elastic modulus of the swollen Emax. The vast difference between the elastic modulus of Emax when printed and when swollen indicates that the absorption of small volumes of water by Emax has a considerable plasticizing effect the mechanical properties of Emax. 
The as-printed composite materials display similar behavior to the swollen composites with increasing elastic modulus, failure strength, failure strain and work of extension corresponding to an increased Emax volume fraction. The experimental values of elastic modulus for the as-printed hydrogel composites were also compared to the rule of mixtures in Figure 8b. In this case the measured elastic moduli fell below the upper bound rule of mixtures values. This discrepancy between experimental and theoretical elastic modulus is likely a result of the Emax phase absorbing water from the adjacent ICE gel phase during the printing process. The theoretical values given in Figure $8 \mathrm{~b}$ use the modulus of fully-dry Emax and may not represent the true modulus for as-printed Emax in the composite samples, since it is shown above that small amounts of water absorption significantly decreases the elastic modulus of Emax. To demonstrate the effect of moisture absorption by Emax from adjacent ICE gel, a 91\% Emax hydrogel composite samples was printed and tested in tension after all of the ICE gel had been carefully removed by a scalpel. The Emax phase of this sample had an elastic modulus of $29 \pm 2 \mathrm{MPa}$, which is appreciably smaller than the elastic modulus of the fully dry Emax material. These results indicate that water can be absorbed by the Emax from adjacent ICE gel, thereby lowering the reinforcing efficiency and overall modulus of the as-printed composites. 

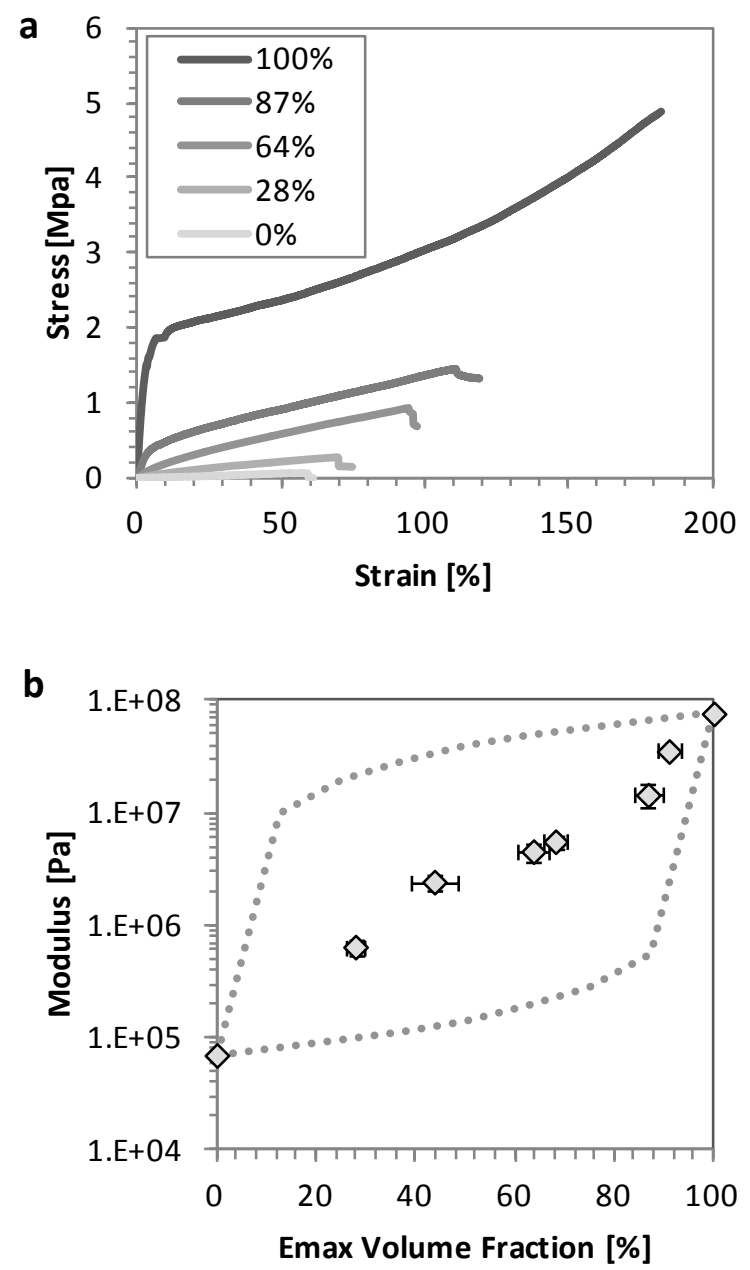

Figure 8. a. Typical stress strain curves for as-printed hydrogel composites with 0, 28, 64, 87 and 100\% Emax volume fractions. b. The elastic modulus for as-printed hydrogel composites as a function of Emax volume fraction including the theoretical upper (Equation 4) and lower (Equation 5) bounds for composite elastic modulus. 
Table 3. Summary of the mechanical properties of as-printed hydrogel composites. ${ }^{\mathrm{c}}$

\begin{tabular}{|c|c|c|c|c|c|c|}
\hline $\begin{array}{l}\text { Emax } \\
\text { Volume } \\
\text { Fraction [\%] }\end{array}$ & $\begin{array}{l}\text { Elastic } \\
\text { Modulus } \\
{[\mathrm{MPa}]}\end{array}$ & $\begin{array}{l}\text { Upper } \\
\text { Bound } \\
\text { [MPa] }\end{array}$ & $\begin{array}{l}\text { Lower } \\
\text { Bound } \\
{[\mathrm{MPa}]}\end{array}$ & $\begin{array}{l}\text { Failure } \\
\text { Stress } \\
{[\mathrm{MPa}]}\end{array}$ & $\begin{array}{l}\text { Failure } \\
\text { Strain } \\
{[\%]}\end{array}$ & $\begin{array}{l}\text { Work of } \\
\text { Extension } \\
{\left[\mathrm{kJ} / \mathrm{m}^{3}\right]}\end{array}$ \\
\hline 0 & $\begin{array}{l}0.070 \\
0.004\end{array}$ & 0.070 & 0.070 & $\begin{array}{l}0.046 \\
0.003\end{array}$ & $62 \pm 7$ & $13 \pm 2$ \\
\hline $28 \pm 2$ & $0.64 \pm 0.09$ & 19 & 0.093 & $\begin{array}{l}0.26 \\
0.01\end{array}$ & $64 \pm 7$ & $90 \pm 20$ \\
\hline $43 \pm 5$ & $2.4 \pm 0.3$ & 29 & 0.11 & $\begin{array}{l}0.71 \\
0.08\end{array}$ & $60 \pm 7$ & $240 \pm 50$ \\
\hline $64 \pm 3$ & $4.4 \pm 0.9$ & 38 & 0.14 & $\begin{array}{l}0.81 \\
0.04\end{array}$ & $90 \pm 10$ & $480 \pm 90$ \\
\hline $68 \pm 2$ & $5.6 \pm 0.7$ & 48 & 0.19 & $1.2 \pm 0.1$ & $96 \pm 6$ & $720 \pm 90$ \\
\hline $87 \pm 3$ & $14 \pm 3$ & 57 & 0.28 & $1.5 \pm 0.1$ & $110 \pm 10$ & $1200 \pm 80$ \\
\hline $91 \pm 2$ & $53 \pm 2$ & 67 & 0.56 & $3.5 \pm 0.2$ & $170 \pm 4$ & $4200 \pm 400$ \\
\hline 100 & $76 \pm 9$ & 76 & 76 & $5.5 \pm 0.4$ & $190 \pm 20$ & $6800 \pm 400$ \\
\hline
\end{tabular}

\footnotetext{
${ }^{\mathrm{C}}$ Upper limit and lower limit indicate elastic modulus calculations using equations 4 and 5, respectively.
}

\section{Fabrication of artificial meniscus}

An artificial meniscus was 3D printed to demonstrate how this fibre reinforced hydrogel printing technique can be adapted to fabricate complex hydrogel structures for real world applications. The meniscus is a crescent shaped cartilage structure in the knee (Figure 9a) that acts to disperse the weight of the body and reduce friction within the knee joint. Meniscal injuries that do not heal and that cannot be repaired are treated by removing the damaged meniscus and replacing it with either an allograft or a synthetic replacement. ${ }^{24} 3 \mathrm{D}$-printing is a valuable tool for fabricating such a synthetic implantable structure because patient specific implants can be easily made by tailoring the digital model of the implant to match the size and shape of an individual patient's knee. Fibre 
reinforced hydrogels are a logical choice of material for use in an artificial meniscus because the natural meniscus is also a fibre reinforced hydrogel. ${ }^{16}$ The meniscus contains a collagen fibre network that is made up of circumferential fibres and radial or 'tie' fibres ${ }^{25}$ (Figure 9b) which give the cartilage strength and stiffness in the desired directions.

To print a fibre reinforced hydrogel artificial meniscus CAD software was used to create a digital model of the meniscus. The simplified digital model of an artificial meniscus contained both circumferential fibres and radial fibres so as to mimic the structure of natural meniscus (Figure 9c). The concentration of Emax was spatially varied throughout the digital model so as to control the mechanical properties in different regions of the artificial meniscus. A low Emax concentration was used around the arc of the meniscus in order to replicate the low modulus of soft tissue. The ends of the meniscus were designed with a high Emax concentration to create a hard site that could be used to attach the artificial meniscus to hard bone. The artificial meniscus was fabricated by printing alternating layers of the circumferential fibres and the radial fibres (Figure 9d-e). A photograph of the completed 3D printed fibre reinforced hydrogel artificial meniscus is shown in Figure 9f. The printed structure was loaded in compression to 500 N (50 kg) without any observable damage. 

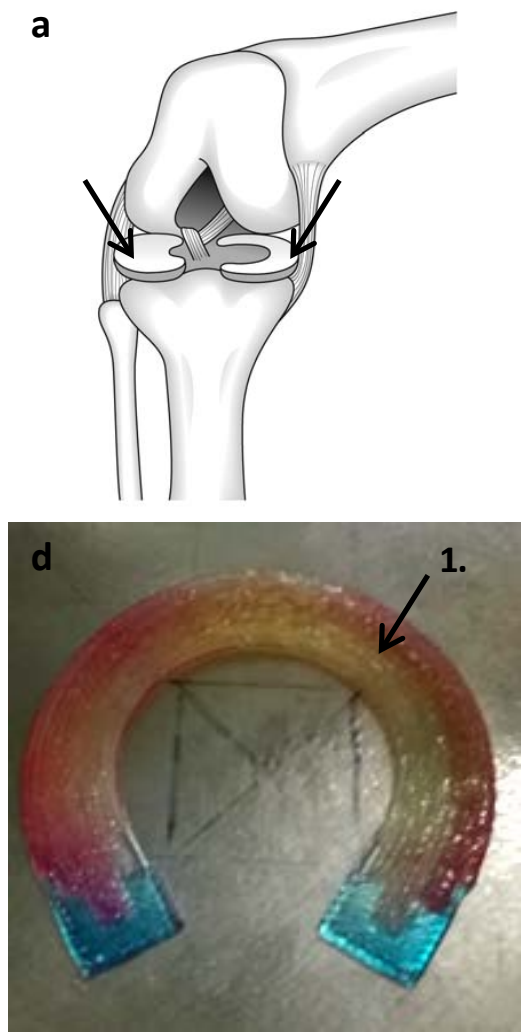

b
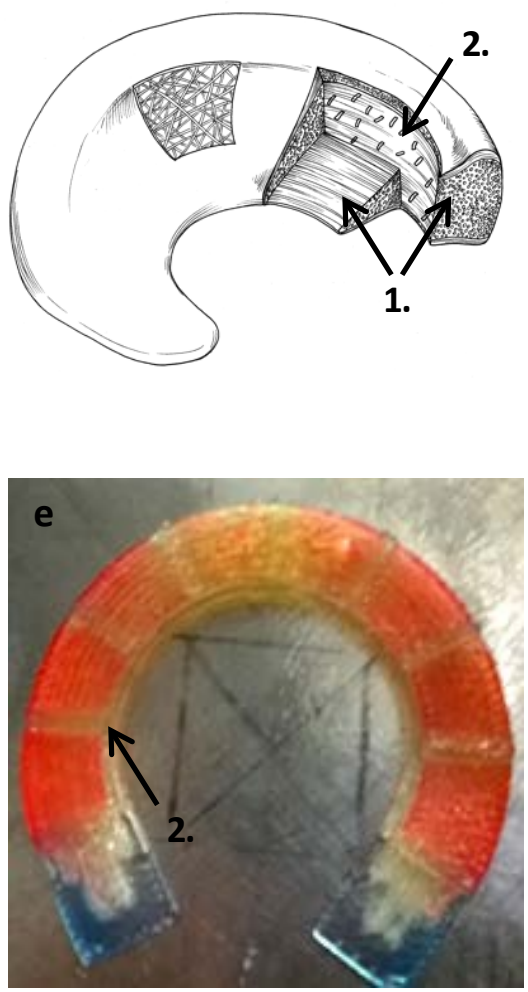

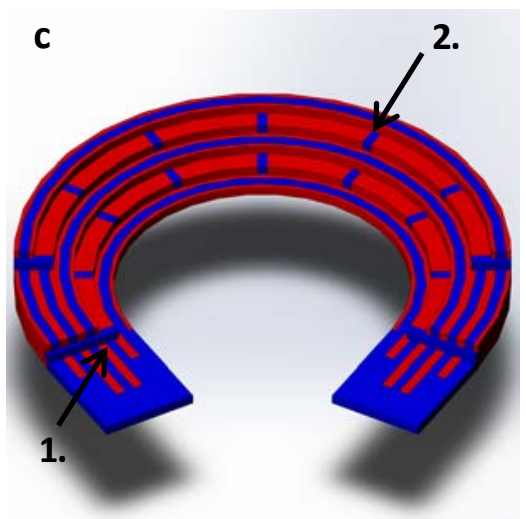

f

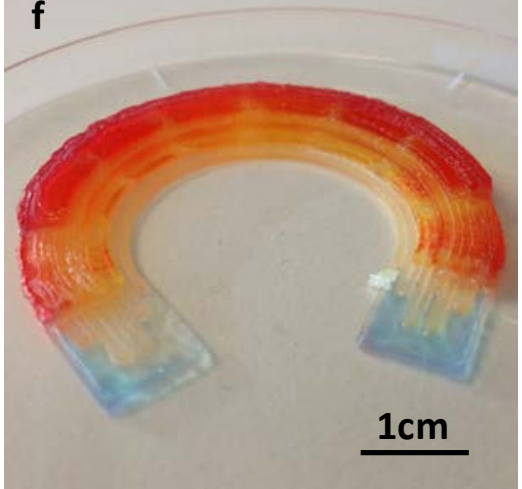

Figure 9. a. A diagram of the knee joint illustrating the shape and position of the meniscus cartilage (arrowed) reproduced from ref. 26. b. An illustration of the meniscus cartilage showing the collagen fiber network reproduced from ref. 27. Label 1 indicates the circumferential fibres and label 2 indicates radial fibres. c. A CAD model of the artificial meniscus. Photographs taken after printing layers of (d) circumferential fibres and (c) radial fibres. f. Completed 3D-printed artificial meniscus.

\section{CONCLUSIONS}

3D printing in conjunction with digital modeling was used to fabricate fibre reinforced hydrogels in a single-step process. By altering the ICE gel and Emax volume fractions of the digital models a full spectrum of soft / wet through to hard / dry composite materials were prepared. When the mechanical characteristics of these printed composites were compared with standard composite theory the swollen fibre reinforced hydrogels were found to adhere to the rule of mixtures. An apparent discrepancy was observed, however, between the as-printed materials and the rule of mixtures estimation of elastic modulus. During the printing process the Emax fibres absorb water from the adjacent ICE gel matrix and absorption of water was shown to drastically reduce the 
elastic modulus of Emax. The discrepancy between the as-printed composites and the rule of mixtures can be accounted for by the softening of the Emax phase by the absorption of water from the adjacent hydrogel.

This study has demonstrated the capability of 3D printing of simple fibre composites based on hydrogel materials. An example application was demonstrated by printing an artificial meniscus cartilage which mimicked the complex 3D shape and incorporated spatially varying fibre reinforcements. Improvements in print resolution when matched to appropriate ink rheology and solidification methods offer the potential to print more complex composites structures, such as particulate reinforced, 3D reinforcement and cellular reinforcements, including honeycomb structures. The further development of these 3D modeling techniques is expected to be useful for the fabrication of multi-component hydrogel structures or devices with multiple applications in micro-fluidics (pumps and valves), robotics (artificial muscles) and bionics (tissue scaffolds and artificial organs).Ultimately, we hope to be able to produce 3D printed versions of soft tissues like tendons, cartilage, skin and muscle where spatial variation in composition and properties is a major contributor to function.

\section{ACKNOWLEDGEMENTS}

The authors acknowledge the Australian National Fabrication Facility for providing access to facilities and the Australian Research Council for funding this work through the Centres of Excellence, Australian Professorial Fellowship (GMS) and Future Fellowship (MihP) programs.

\section{AUTHOR INFORMATION}

\section{Corresponding Author}

*E-mail: gspinks@uow.edu.au

\section{REFERENCES}


(1) Jones, N., The Print Revolution Three-Dimensional Printers are Opening up New Worlds to Research. Nature 2012, 487, 22-23.

(2) Espalin, D.; Muse, D.; MacDonald, E.; Wicker, R., 3D Printing Multifunctionality: Structures with Electronics. Int. J. Adv. Manuf. Technol. 2014, 72, 963-978.

(3) Villar, G.; Graham, A. D.; Bayley, H., A Tissue-Like Printed Material. Science 2013, 340, 48-52.

(4) Khatiwala, C.; Law, R.; Shepherd, B.; Dorfman, S.; Csete, M., 3D Cell Bioprinting for Regenerative Medicine Research and Therapies. Gene Ther. Regul. 2012, 07, 1-19

(5) Symes, M.D., Kitson, P.J., Yan, J., Richmond, C.J., Cooper, G.J.T., Bowman, R.W. Vilbrandt, T. and Cronin, L., Integrated 3D-printed reactionware for chemical synthesis and analysis. Nature Chem. 2012, 4, 349-354.

(6) Fan, H.; Yu, Y.; Stump, A.; Reed, S. T.; Baer, T.; Schunk, R.; Perez-Luna, V; Lopez, G. P.; Brinker, C. J., Rapid Prototyping of Patterned Functional Nanostructures. Nature 2000, 405, 56-60.

(7) Zhou, M. Y.; Xi, J. T.; Yan, J. Q., Modeling and Processing of Functionally Graded Materials for Rapid Prototyping. J. Mater. Process. Tech. 2004, 146, 396-402.

(8) Ferris, C. J.; Gilmore, K. G.; Wallace, G. G.; in het Panhuis, M., Biofabrication: An Overview of the Approaches used for Printing of Living Cells. Appl. Microbiol. Biotechnol. 2013, 97, 4243-4258.

(9) Murphy, S. V.; Skardal, A.; Atala, A., Evaluation of Hydrogels for Bio-Printing Applications. J. Biomed. Mater. Res., Part A 2013, 101A, 272-284.

(10) Melchels, F. P. W.; Domingos, M. A. N.; Klein, T. J.; Malda, J.; Bartolo, P. J.; Hutmacher, D. W., Additive Manufacturing of Tissues and Organs. Prog. Polym. Sc. 2012, 37, 1079-1104. 
(11) Derby, B., Printing and Prototyping of Tissues and Scaffolds. Science 2012, 338, 921-926.

(12) Billiet, T.; Vandenhaute, M.; Schelfhout, J.; Van Vlierberghe, S.; Dubruel, P., A Review of Trends and Limitations in Hydrogel-Rapid Prototyping for Tissue Engineering. Biomaterials 2012, 33, 6020-6041.

(13) Pataky, K.; Braschler, T.; Negro, A.; Renaud, P.; Lutolf, M. P.; Brugger, J., Microdrop Printing of Hydrogel Bioinks Into 3D Tissue-Like Geometries. Adv. Mate. 2012, 24, 391-396.

(14) Bakarich, S. E.; in het Panhuis, M.; Beirne, S.; Wallace, G. G.; Spinks, G. M., Extrusion Printing of Ionic-Covalent Entanglement Hydrogels with High Toughness. J. Mate. Chem. B 2013, 1, 4939-4946.

(15) Muroi, H.; Hidema, R.; Gong, J.; Furukawa, H., Development of Optical 3D Gel Printer for Fabricating Free-Form Soft and Wet Industrial Materials and Evaluation of Printed Double-Network Gels. J. Solid Mech. Mater. Eng. 2013, 7, 163-168.

(16) Agrawal, A.; Rahbar, N.; Calvert, P. D., Strong Fiber-Reinforced Hydrogel. Acta Biomater.2013, 9, 5313-5318.

(17) Lin, S.; Cao, C.; Wang, Q.; Gonzalez, M.; Dolbow, J. E.; Zhao, X., Design Of Stiff, Tough and Stretchy Hydrogel Composites via Nanoscale Hybrid Crosslinking and Macroscale Fiber Reinforcement. Soft Matter 2014, DOI: 10.1039/c4sm01039f.

(18) Seo, Y.; Youn, H.; Park, C.; Song, K.; Park, J., Reinforced Bioartificial Dermis Constructed with Collagen Threads. Biotechnol. Bioprocess Eng. 2008, 13, 745-751.

(19) Etches, J. A.; Bond, I. P., Development of a Self-Actuating Fibre Reinforced Ionic Epoxy Gel Polymer Composite. Smart Mater. and Struct. 2011, 20, 1-8. 
(20) Liao, I. C.; Moutos, F. T.; Estes, B. T.; Zhao, X.; Guilak, F., Composite Three-Dimensional Woven Scaffolds with Interpenetrating Network Hydrogels to Create Functional Synthetic Articular Cartilage. Adv. Funct. Mater. 2013, 23, 5833-5839.

(21) Calvert, P., Hydrogels for Soft Machines. Adv. Mater. 2009, 21, 743-756.

(22) Mezger, T. C., The Rheology Handbook, 1st ed; Vincentz Network: Hannover, 2006.

(23) Hull, D., An Introduction to Composite Materials, 1st ed; The Press Syndicate of the University of Cambridge: Cambridge, 1981.

(24) Linder-Ganz, E.; Elsner, J. J.; Danino, A.; Guilak, F; Shterling, A., A Novel Approach for Evaluating Contact Mechanics of Meniscal Replacements. J. Biomech. Eng. 2010, 132, 1-6

(25) Bullough, P. G.; Munuera, L.; Murphy, J.; Weinstein, A. M., The Strength of the Menisci of the Knee as it Relates to their Fine Structure. J. Bone Joint Surg. 1970, 52, 564-570.

(26) Hauser, R. A.; Phillips, H. J.; Maddela, H. S., The Case for Utilizing Prolotherapy as FirstLine Treatment for Meniscal Pathology: A Retrospective Study Shows Prolotherapy is Effective in the Treatment of MRI-Documented Meniscal Tears and Degeneration. J. Prolotherapy 2010, 2, 416437.

(27) Greis, P. E.; Bardana, D. D.; Holmstrom, M. C.; Burks, R. T., Meniscal Injury: I. Basic Science and Evaluation. J. Am. Acad. Orthop. Sur. 2002 10, 168-176. 


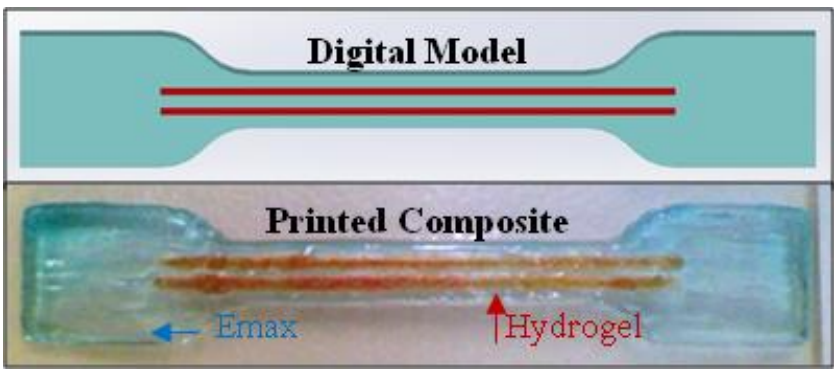

